\title{
MEDIOS CIUDADANOS Y BIG DATA: LA EMERGENCIA DEL ACTIVISMO DE DATOS
}

\section{CITIZENS' MEDIA MEETS BIG DATA: THE EMERGENCE OF DATA ACTIVISM}

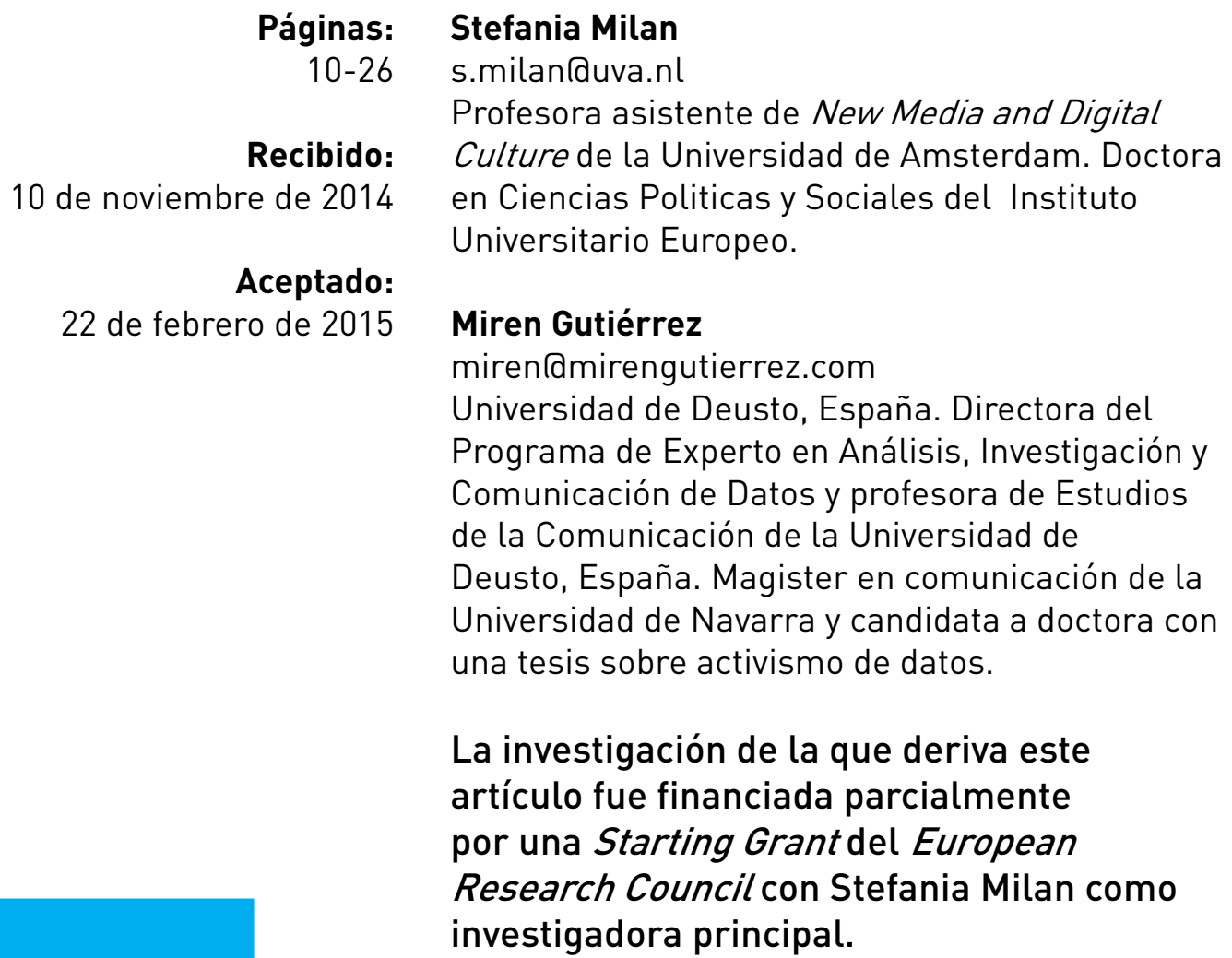

La investigación de la que deriva este artículo fue financiada parcialmente por una Starting Grant del European Research Council con Stefania Milan como investigadora principal.

Resumen

Los big data representan nuevos retos y nuevas oportunidades para la ciudadanía. Las prácticas del "activismo de datos" surgen de la intersección de las dimensiones social

y tecnológica de la acción humana, por la cual la ciudadanía adopta una postura crítica hacia los big data, que se apropia y manipula para hacer campaña y promover el cambio social. Este artículo teórico explora el surgimiento del activismo de datos como una realidad empírica y una herramienta heurística para estudiar cómo la gente se relaciona políticamente con los big data. Ponemos en contexto este concepto a través de una revisión de literatura académica y ofrecemos una definición del activismo de datos, así como una agenda tentativa para su estudio. Argumentamos que el activismo de datos representa una nueva forma de medio ciudadano que coloca en su mismo centro una aproximación crítica hacia los big data.

Palabras clave

Big data, activismo de datos, ciudadanía,
cambio social.

Big data, activismo de datos, ciudadanía,
cambio social.

\section{Abstract}

Big data presents citizens with new challenges and opportunities. 'Data activism' practices

emerge at the intersection of the social and

techsnological dimension of human action, whereby citizens take a critical approach to big data, and appropriate and manipulate data for advocacy and social change. This theoretical article explores the emergence of data activism as an empirical reality and a heuristic tool to study how people engage politically with big data. We ground the concept on a multidisciplinary literature review, and offer a situated definition of data activism and a tentative agenda for its study. We argue that data activism represents a new form of citizens' media that has a critical approach to big data at its core.

\section{Key words}

Big data, data activism, citizienship, social change. 
"Vivimos una época en la que estamos rodeados de datos. Los gobiernos de todo el mundo están abriendo sus almacenes de datos y permitiendo que cualquiera acceda a ellos", explica Simon Rogers, editor del Guardian Datablog. Efectivamente, estamos viviendo tiempos de abundancia de datos. Enormes bases de datos se generan continuamente y son automáticamente recogidas y depositadas gracias a una variedad de tecnologías, como sensores aéreos, ubicuos aparatos móviles, y lectores de identificación por radiofrecuencia. Mientras navegamos por el ciberespacio, nuestras ac tividades dejan huellas de lo que hacemos en una miríada de metadata y registros almacenados por los proveedores de servicios de comunicación. La habilidad de generar cada vez más datos y darles sentido ha llevado a estudiosos a habla historia de la humanidad que Hellerstein (2008) ha llamado "la revolución industrial de los datos".

Lo que se conoce como big data incluye, por ejemplo, las diversas bases de datos generadas por agencias gubernamentales publicadas, a veces, como "datos abiertos" (i.e. datos que la ciudadanía puede usar, reusar y redistribuir) Big data se refiere también a la extraordinaria cantidad de documentos en video y audio, texto, enlaces y etiquetas que resultan de la distribución y el almacenamiento online, y la información generada por la interacción humana en las plataformas de las redes sociales. Big data son también los procesos de indexación de las actividades en internet y los metadata resultantes de la vigilancia digital de los gobiernos. Finalmente, son el resultado del proceso de "datificación", es decir, "la habilidad de convertir en datos muchos aspectos de la realidad que nunca habían sido cuantificados antes" (Cukier y Mayer-Schoenberger, 2013, pp. 28-40), como las amistades en forma de "me gusta".
Se espera que la tecnología y el mercado de los en 2011, a USD 23.800 en 2016, con "una tasa de crecimiento anual del treinta por ciento, lo que equivale a unas siete veces el mercado de la tecnología de la información y la comunicación", (Vesset, 2012). No sorprende, pues, que el analista Abhishek Mehta (2011) vea los datos como fundamental materia prima para una variedad de sistemas de negocio socioeo la meteorología las finanzas y la genómica se han enfrentado lesde con con el reto de dar sentido a grandes bases de datos, la escala sin precedentes de la información generada hoy propone desafíos completamente nuevos a una serie de profesiones que tratan con información. El periodismo, como visibilizar in grandes audiencias, está en la vanguardia (de esta "revolución de los datos".

Los big data no solo implican nuevos retos; también, nuevas oportunidades. Rogers dice de nuevo: "esto es muy trascendente porque significa que tenemos acceso a datos a los que nunca habríamos accedido en el pasado. Nos ofrece relatos nuevos, nuevas formas de ver el mundo" (Bonechi, 2012). La ciudadanía se ha dado cuenta del potencial de los datos para promover el cambio social. Y de esta nueva conciencia surgen nuevas prácticas sociales enraizadas en la tecnología y los datos, a las que hemos llamado "activismo de datos".

El activismo de datos implica nuevas prácticas sociales que adoptan una actitud crítica hacia los big data. Ejemplos de activismo de datos incluyen el mapeo colectivo y la georreferenciación de mensajes de víctimas de desastres para facilitar las operaciones humanitarias 0 la elaboración de datos abiertos para hacer campaña o incidencia política. Sin embargo, el activismo de datos también abarca tácticas de resiste la intrusión y recolección masiva de datos a cargo de empresas privadas y gobiernos, como la encriptación de las comunicaciones privadas o los métodos disruptivos para obstaculizar la maquina de recolección de datos.

El activismo de datos es un constructo teórico basado en observaciones empíricas. Lo proponemos como una herramienta heurística útil para explorar cómo la gente se involucra polí ticamente con los big data y la recolección masiva de datos. En este artículo teórico, exploramos el potencial de los datos, de las narrativas basadas en datos, y del software para recabar y analizar datos como herramientas de campaña para el activismo y el cambio social. A continuación, presentamos el contexto político y socioeconómico donde el activismo de datos ha emergido y ofrecemos una definición preliminar. Más adelante, hacemos una revisión de literatura académica multidisciplinar para ponerlo en contexto y explorar algunas de sus facetas. Finalmente, ofrecemos una definición revisada para el activismo de datos y proporcionamos una agenda tentativa para el estudio de este fenómeno.

\section{Configuración de \\ la escena: estados \\ informacionales y política computacional}

Desde 2012, la organización mediática sin ánide documentos clasificados, incluyendo cables diplomáticos estadounidenses e informes de campo de las guerras de Afganistán e Iraq (c.f. Brevini et al., 2013). Así mismo, los documentos clasificados como secretos que Edwards J. Snowden, el consultor estadounidense experto en inteligencia, ofreció a varios medios de co- municación destaparon las estrategias de acopio de datos masivo que efectúan las agencias de seguridad nacional.

Estas revelaciones provocaron agitación pública y forzaron a las autoridades a reaccionar. Las revelaciones de Snowden movilizaron jefes de Estado e incluso fueron discutidas en la Asamblea General de Naciones Unidas. Los cables filtrados por WikiLeaks tuvieron consecuencias sideran el catalizador de la revuelta de Túnez de 2010-2011. Algunos incluso afirman que "podemos contar Túnez como la primera vez que WikiLeaks ha empujado a la gente hasta el borde del precipicio” (Dickinson, 2011).

El caso de WikiLeaks demuestra el crecimiento en magnitud y complejidad que la información ha cambiado la naturaleza del Estado Nación, así como su relación con la ciudadanía. Los gobiernos "deliberada, explicita y consistentemente controlan la generación, proceso y canalización de la información, y lo usan para ejercer su poder" (Braman, 2009, p. 1). En opinión de Braman, el estado se ha convertido en un estado "informacional": en otras palabras, este se caracteriza por "los cambios en la naturaleza del poder y su ejercicio a través de la política de la información” (Ibíd.). Pero hay más. De acuerdo con Tufekci, las tecnologías digitales "han dado paso a una nueva combinación de big data y prácticas computaciones que permiten la recopilación masiva de datos latentes y un modelo computacional complejo, lo que aumenta la capacidad de aquellos con recursos y acceso para usar estas herramientas a fin de llevar a cabo campañas de persuasión e ingeniería social en círculos políticos, cívicos y comerciales que son altamente efectivas, opacas e impenetrables" (Tufekci, 2014). El surgimiento de esta "política computacional" está intimamente ligado a "la emergencia de los big data, el cambio de enfoque de lo demográfico a 
lo individual, la opacidad y el poder del modelo computacional, el uso de la ciencia del comportamiento persuasivo, la experimentación dinámica en tiempo real que los medios digitales hacen posible, y el crecimiento de nuevos agentes que poseen los datos o los medios sociales” (Ibíd.).

Sin embargo, un Estado informacional requiere "colaboración corporativa" (MacKinnon, 2012) para poder ejercer 2012) para poder ejercer sus leyes y reglas: como el caso de las revelaciones de Snowden
pone en evidencia muchas cosas, los gobierpone en evidencia muchas cosas, los gobier-
nos cada vez más se apoyan en "entidades del sector privado como agentes reguladores, usando centros de poder privado para propó sitos estatales” (Braman, 2009, p. 34). Más allá de contribuir a formas de control, los actores corporativos están detrás de un internet con cada vez más aplicaciones, donde artefactos "atados" (que pueden ser modificados solo por sus creadores) terminan poniendo en peligro las cualidades generativas de la red o su capacidad de encontrar espacios fuera del control del gobierno al nivel de usuario (Zittrain, 2008). Más aun, la gente a la que se le hurtan esas capacidades acaba pagando el precio más alto, ya que la vigilancia online sirve para discriminar contra "los miembros de las comundades más crónicamente desatendidas" (Gangadharan, 2012).

El acceso a los datos es poder. Los datos y la información se han convertido en una moneda de cambio cada vez más importante en la políde WikiLeaks y de Wileaks y Snowden. Pero esa misma teccologia que permite a los gobiernos y corporaciones amasar y explotar datos digitales sobre la ciudadanía, ahora ofrece a esa misma ciudadanía la posibilidad de hacer lo opuesto: enfocar y atacar los sistemas digitales y los datos de gobiernos y empresas. De acuerdo con Deibert (2010), WikiLeaks fue solo "un síntoma de una tendencia mucho mayor" [...] "los medios para espiar en el ciberespacio se han expandido dramáticamente por el cambio experimentado en las infraestructuras de comunicación y los hábitos en las redes sociales. [...] El ciberespacio nos ha traído una plétora de servicios de espionaje digital 'hazlo tú mismo’”.

En este escenario, la emergencia de prácticas de base ha llevado a una ciudadanía progresista al centro de la "revolución de los datos".

\section{Definición}

\section{del activismo de datos}

El activismo de datos emerge en la periferia de la sociedad, en el ámbito típicamente asociado con el activismo de base y el compromiso cívico. Sin embargo, está rápidamente evolucionando desde una forma de activismo periférico, elitista y basado en habilidades técnicas hacia una forma de activismo más difuso, de forma que usuarios con menos habilidades participan también. Incluye una serie de prácticas en la intersección de las dimensiones tecnológicas y sociales de la acción humana, con dos objetivos: 0 bien resistir la recolección masiva de datos o bien utilizar la explotación de los datos disponibles para el cambio social.

Para nuestros propósitos analíticos, hemos identificado dos subáreas del activismo de datos: la reactiva y la proactiva. El activismo de datos reactivo incluye las prácticas de resistencia ante las amenazas contra los derechos humanos y civiles que representa la intrusión hubanos y civiles que representa la intrusion datos proactivo implica a aquellos individu y organizaciones de la sociedad civil que se aprovechan de las posibilidades que ofrecen los big data para el cambio social y el compromiso cívico. Tanto el activismo de datos reactivo como el proactivo constituyen dos facetas del mismo fenómeno, que tiene los datos y la información en su núcleo. Son posibles gracias al software que permite desde la manipulación de los datos a la protección de las interacciones online privadas, frente a la recolección automatizada de datos y la intrusión de terceras partes.

La noción del activismo de datos representa una innovación conceptual que se sitúa en la interseccion la intersección de un proceso sociológico (la organización colectiva para actuar), una actividad cognitiva (dar sentido a información compleja), y una práctica sociotécnica (la tecnología, es decir, el software para manipular datos, es central para el activismo da datos) Por tanto, estudiar este fenómeno empírico llama a aplicar una perspectiva interdisciplinaria que sea capaz de tener en cuenta su naturaleza multidimensional. En la próxima sección, revisamos las contribuciones aportadas por una variedad de campos de investigación los estudios de los medios, de los movimientos sociales, del periodismo y de las relaciones internacionales, con el fin de ubicar la noción del activismo de datos en la literatura académica existente.

\section{Una perspectiva caleidoscópica del activismo de datos desde disciplinas diferentes}

El activismo de datos es un fenómeno empíri co emergente para el que tenemos que desarrollar un nuevo vocabulario de conceptos y mecanismos interdisciplinares. ¿Cómo estudiamos un fenómeno social que está enraizado en la tecnología de la comunicación (y la información) que está posicionado entre las dimensiones sociales y comunicativas de la acción humana? Aquí ofrecemos una revisión de conceptos útiles que emergen de ciales y las humanidades. Los conceptos que hemos inspeccionado sirven el propósito de iluminar diferentes aspectos del activismo datos que nos permitirán refinar nuestra noción a la vista de la literatura académica ${ }^{1}$.

Desde los estudios de medios de información (alternativos): medios ciudadanos y empoderamiento

El activismo de datos marca un cambio de perspectiva y actitud hacia la recolección dependientes que han puesto a la ciudadanía

Durante los últimos años, la academia ha propuesto diferentes etiquetas para describir los dicales (Don (Rodriguez, 2001), medios alternativos (Atton, 2001), medios tácticos (Garcia y Lovink, 1997), medios autónomos (Langlois y Dubois, 2005), y muchos más. Hackett y Carroll (2006) se refieren a "prácticas de comunicación de oposición" que emanan de la realidad y están empeñadas en su cambio, buscando cultivar esferas públicas alternativas. El experto en medios al-

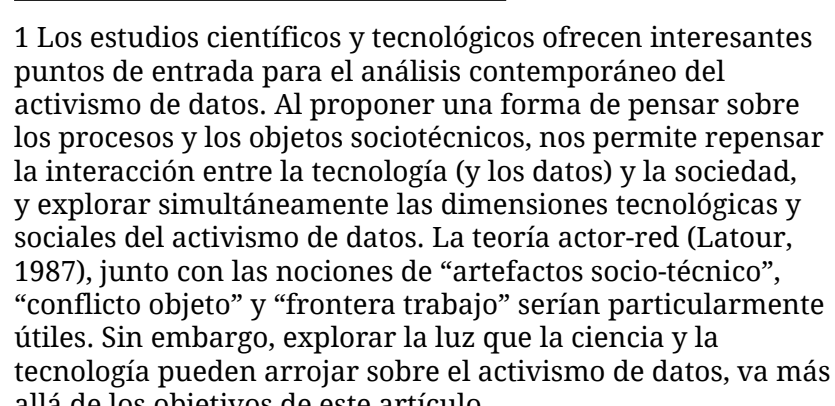
útiles. Sin embargo, explorar la luz que la ciencia y la
ecnnologia pueden arrojar sobre el activismo de datos, va más
allá de los objetivos de este artículo 
ternativos, John Downing, definió los medios radicales como "medios, generalmente de pequeña escala y de formatos muy variados, que expresan una visión alternativa y diferente de la política, prioridades y perspectivas hegemónicas" (Downing, 2001, v-ix). Sin embargo, es el concepto de medios ciudadanos el que ofrece una visión más interesante para el estudio del activismo de datos.

Para este objeto de estudio, lo que hace la noción de los medios ciudadanos tan apropiada es su enfoque en el empoderamiento y en la política de la vida cotidiana. Dentro de la esfera de la comunicación, el empoderamiento es el proceso por el cual individuos y grupos, al participar activamente en las acciones que dan forma a los procesos comunicativos, ejercitan control sobre sus recursos de com ejercitan control sobre sus recursos de comunicación y mensajes. En este ejercicio activo de control sobre las tecnologías, es que la gente incluidos los no expertos, resulta empoderada. Igualmente, Rodríguez ve los medios ciudadanos como un espacio donde la gente pone en práctica su hacer democrático, más allá del tradicional ejercicio del voto (Rodríguez 2001). Además, argumenta que "estas prácticas y estrategias de resistencia constituyen la política de lo cotidiano". Expanden y multiplican los espacios para la acción política, que no está confinada a espacios institucionales sino inserta en la vida social y las interacciones entre iguales (p. 21).

Es interesante que Rodríguez se base en las teorías sobre la democracia radical y el feminismo para definir los medios ciudadanos. En sus palabras, los medios ciudadanos implican
que, primero,

[... ]una colectividad está poniendo en práctica su ciudadanía para activamente intervenir y transformar el panorama mediático; segundo, estos medios es- tán protestando contra códigos sociales, identidades legitimadas y relaciones sociales institucionalizadas; y tercero, estas prácticas de comunicación están empoderando a la comunidad involucrada hasta el punto que estas transformaciones y cambios se hacen posibles (Rodríguez 2001, p. 20).

Todo lo anterior vale para hablar del activismo de datos también, pero con salvedades. Aunque el espíritu de protesta y el reto que plantea a las identidades establecidas y relaciones sociales es común en ambos, el periodismo de datos se distancia de los medios ciudadanos dividuales. Existe dentro del ámbito del activismo de datos una tensión nueva entre dividuo y la dima tensión nueva entre el individuo y la dimensión colectiva de la acción organizada que amenaza con arrinconar los términos de referencia de la comunidad que son tan centrales en la definición de los medios ciudadanos, comunitarios y alternativos.

\section{Desde los estudios de}

\section{periodismo: el periodismo se}

da de bruces con los datos ly el cambio social)

En la primavera del 2010, WikiLeaks publica un video titulado "Asesinato colateral", donde se ve un helicóptero de los Estados Unidos que está disparando contra civiles iraquíes. El furioso debate, surgido como consecuencia de la revelación, forzó al gobierno norteamericano a reaccionar. Durante los últimos años, las revelaciones de WikiLeaks han ofrecido una cantidad de materia prima para el periodismo de investigación sin precedentes, que resultó en una alianza entre cinco prominentes medios de información, incluyendo El Pais y el New York Times. Dan Gillmor (2005), uno de los representantes más destacados del periodis- mo que toma partido, declaró: "quizás sea el momento de otorgar una cariñosa despedida al viejo canon del periodismo: la objetividad. Pero nunca nos desharemos de los valores principios en los que está anclado”.

Observar WikiLeaks y la trayectoria reciente del periodismo ofrece algunas ideas sobre el activismo de datos. En particular, tres géneros, hablan directamente de la práctica del activismo de datos: el periodismo de investigación, el periodismo de campaña y los medios ciudadanos. El periodismo de investigación es considerado el mejor tipo de periodismo. Implica un periodismo de asuntos que conciernen a la ciudadanía "cuando otros están tratando de esconderlos del público" (Northmore, 2001). El periodismo de campaña deliberadamente adopta una perspectiva partisana en su informar. Está basado en hechos, pero adopta abiertamente una postura (Careless, 2000), dado que los periodistas de campaña piensan que el público está en mejor servicio con diversidad y transparencia.

Desde los noventa, internet ha revolucionado la práctica periodística y "ha reventado por las la práctica pe costuras el sistema de puertas y porteros”2 típico de los medios tradicionales (Rosen, 2006). Como resultado de ellos, surgieron los medios ciudadanos, que abrieron las puertas de la redacción a los no profesionales. De acuerdo con Rosen (2008), vemos los medios ciudadanos en acción "cuando la gente que antes conocíamos como audiencia emplea las herramientas a su alcance para informarse mutuamente”. Esta práctica tiene conexión con lo que otros han llamado periodismo alternativo (Atton y Hamilton, 2009).

La llegada de los big data ha significado una evolución para el periodismo también, ejemplificada por el "periodismo de datos". El pe-

2 Traducción literal de gates y gatekeeers riodismo de datos usa los avances de la ciencia social y los métodos computacionales para buscar relatos y noticias en complejas bases de datos. Representa una evolución del periodismo de precisión, porque este periodismo usa metodologías de investigación avanzadas para elevar el nivel de rigor del periodismo más allá de la anécdota (Meyer, 2002). En El cuarto poder en red, Víctor Sampedro (2014) argumenta que las nuevas prácticas periodísticas, enraizadas en la tecnología y los datos, son el comienzo de un innovador y mejor periodismo. Cita a WikiLeaks y Snowden como ejemplos de cómo poner en práctica un prototipo de medios críticos e independientes para el bien común. En su opinión, hackers y whistleblowers $^{3}$ han vuelto a recuperar los ideales del viejo periodismo de investigación. "El viaje que proponen los hacktivistas es de ida y vuelta: el periodismo que viene es el que vuelve a sus orígenes. Hay que refundar los medios para que recobren sus fines. Porque se trata de eso: que retomen los objetivos que dieron legitimidad democrática a esta profesión”. El activismo de datos reclama cosas similares.

El periodismo de datos ha ofrecido un soplo de aire fresco a una profesión sumergida en una profunda crisis, que se enfrenta a una generalizada y continua caída en circulación, lectoría, audiencias, finanzas, e incluso credibilidad. Si el periodismo se halla "en estado de sitio", el advenimiento del periodismo de datos resulta de particular relevancia, ya que señala también una evolución en la función (social) de los periodistas: "usando datos, el papel de los periodistas cambia su foco principal: de ser los primeros en informar a ser aquellos que nos explican el significado de un suceso concreto" (Gray et al., 2012, p. 4). Y mientras los perio3 Nuestro idioma no parece tener una adecuada traducción de
este vocablo inglés, ya que palabras como 'delator', 'soplón' o este vocablo inglés, ya que palabras como 'delator', 'soplón'? 0
'chivato' tienen connotaciones negativas. Entretanto, vocablos como hacker parecen haber sido absorbidos sin problemas por 
distas usan datos, progresivamente pasan de datos estaba el movimiento. El 99 por ciento la observación al análisis, la denuncia, la concienciacion cienciación y las recomendaciones. Ese es

La relación de los periodistas con los datos ha forjado alianzas sin precedentes, que son de enorme importancia para los observadores del activismo de datos. Por ejemplo, la red de base Hacks/Hackers (hackshackers.com) cone a periodistas (hacks) con tecnólogos conecta a periodistas (hacks) con tecnólogo (hackers), facilitando el intercambio de habilidades entre los dos grupos. Una variedad de plataformas que facilitan la tarea de navegar y dar sentido a los datos proporcionan las conexiones y a la colaboración entre el profesional de la información y la ciudadanía con interés por su realidad social. Por ejemplo, Open Knowledge Foundation ha desarrollado el portal de datos abiertos CKAN (ckan.org), que es utilizado para explorar bases de datos abiertas por los gobiernos; y OpenSpending (openspending.org) permite a no expertos explorar más de trece millones de transacciones financieras gubernamentales de sesenta y seis países.

\section{Desde los estudios de los \\ movimientos sociales: \\ identidades técnicas y}

comunidades informales

Septiembre de 2011. Los manifestantes anpor la policía Se refugian por zuccoti. y asi empieza el campano Parque Zuccotti, y así empieza el campamento que pondrá en marcha la protesta global conocida como “Occupy Wall Street”. El eslogan de Occupy (Somos el 99\%) llama la atención de ese 99 por ciento de la población: los destiene acceso a recursos como prédito. "Fue reache a rio contra el 1 por ciento es un cálculo de datos", argumenta Simon Rogers, editor del Guardian Datablog. Los manifestantes del Occupy "han conseguido usar los datos para mostrar qué está pasando en el mundo".

Como muestran las protestas Occupy, la sociedad civil organizada recurre cada vez más al evocaprovocar el compromiso cívico y la protesta, y para hacer incidencia. Pero, ¿cómo usan los movimientos sociales los datos para el cambio social? Los activistas de datos se han posicionado como intérpretes de los datos, actuando como facilitadores en la esfera pública contemporánea, rica en datos. Se aprovechan de la producción descentralizada y colaborativa, y del capital humano dis

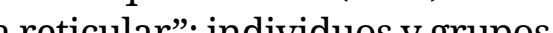
que comparten su experiencia en desar sos software y análisis de datos para crear o hacer accesibles a otros herramientas de análisis y plataformas de datos.

Mientras que el activismo de datos es relativamente desconocido en la academia, las fuerzas sociales que tiene por detrás no son nuevas. Los do de las tecnologías de comunicación por lo menos desde los cincuenta, tanto para expresarse como para propiciar el cambio social: es lo que se ha llamado "medios de campaña”, o el uso creativo y táctico de los medios para generar cambios sociales (Milan y Padovani, 2014). Así mismo, varias corientes sociani, 2014). Asi mismo, varias corrientes sociales y subculturas influyen sobre sobre el activismo de datos actual: por ejemplo, la cultura hacker de los setenta y más adelante; los activistas reforcompromiso técnico como forma de oposición frente al conocimiento elitista (Dunbar-Hester, 2014); el activismo radical tecnológico de activistas han reprogramado y se han apropiamistas estadounidenses que abogaron por un los derechos digitales que se involucraron en la resisten actitud "hazlo tú mismo" de los hackerspaces, hacklabs y makerspaces (Maxigas, 2014), y los movimientos orientados a productos como la cultura de las fuentes abiertas (Hess, 2005). Los activistas de datos han tomado prestados de estas subculturas el énfasis en el activismo emprendedor y la noción del compartir, la colaboración entre colegas, el acceso a la información, la manipulación y el juego con código, y la mejora del mundo a través de respuestas tecnológicas. Revisar de cerca estos estudios proporcionará datos interesantes para interpretar a los activistas de datos de hoy y su impacto en la sociedad en su conjunto.

En Social Movements and their Technologies, Milan (2013b) explora las interacciones entre los movimientos sociales y sus "tecnologías liberadas" y se centra en la irrupción de proyectos de internet radicales (prácticas de comunicación emancipadoras) como sujeto político. Explica cómo los grupos tecnológicos radicales, que crearon alternativas autónomas y clandestinas a los sistemas de comunicación manera la gente se comunica en la vida real. Del mismo modo, los activistas de datos se relacionan en prácticas que cambiarán la forma como los activistas harán campaña y los medios (incluidos los de los periodistas ciudadanos) diseminarán información en el futuro. Ambos contribuyen a los valores progresistas y a los valores de la sociedad donde vivimos, ya que sus sistemas de valor y sus modelos organizacionales enfatizan la creación y los procesos de decisión colectivos, abiertos y descentralizados.

Dunbar-Hester (2012) ha estudiado la radio de baja potencia en los Estados Unidos, centrándose en particular en los grupos que promueven una relación crítica con la tecnología (por ejemplo, construyendo su propio radiotransmisor). Esta estudiosa señala el surgimiento en estas comunidades de una "identidad técnica" que se basa en la disposición proactiva que os activistas desarrollan al ejercer su activismo mediado por la tecnología. Sus integrantes usan esa identidad técnica para activamente "marcar las diferencias entre su grupo y otros en el terreno del trabajo de los medios democráticos” (Dunbar-Hester, 2012, p. 149).

Podemos aprender algo del activismo de datos a partir de la literatura académica sobre los movimientos científicos e intelectuales que han emergido desde la ciencia y los estudios de la tecnología, y de sus intersecciones con los estudios de los movimientos sociales. Los activistas de la tecnología y los movimientos orientados a un producto (como el activismo de código fuente abierto) son inherentemente políticos, y sus prácticas buscan alterar la distribución del poder (Frikel y Gross, 2005). Se involucran en el "desarrollo de formas nuevas 0 alternativas de cultura material" (Hess, 2005). Ocasionalmente, pueden establecer conexiones con empresas del sector privado, que ven como potenciales aliadas en la promoción pén la misma forma, los activistas de datos pueden aliarse con empresas de software alternativas pare con mover software alternativas para pro-

Finalmente, hay mucho que aprender de los recientes estudios de la acción colectiva mediada por la tecnología, en la que los activistas usan las tecnologías de la comunicación electrónica para comunicar, hacer incidencia política, construir comunicadas, organizarse y coordinar acciones. Pero también de las formas radicales de activismo en internet, como el ciberactivismo y el hacktivismo. Existe una naciente literatura sobre este asunto, así que destacaremos solo las contribuciones que parecen ser más útiles para entender el activis- 
mo de datos. Por ejemplo, Bennett y Segerberg (2012) crearon la opción de "acción conectiva", que se refiere a la mediación de las tecnologías digitales en la promoción de interacciones personalizadas, enfatizando las conexiones entre los usuarios individuales, antes que aquellas entre organizaciones y movimientos. Hemos observado esta dinámica también en las formas más radicales del activismo de internet (c. Milan y Hintz 2013), así como en el activismo de datos.

Más similitudes existen entre los activistas de datos de hoy y el activismo radical de internet, es decir, aquellos grupos que explotan la infraestructura técnica de la red en favor de cambio social (Milan, 2013b). Para ambos, la tecnología es simultáneamente la forma de ciedad y un lugar de lucha por propio derecho, en reconocimiento de las relaciones políticas y de poder que son inherentes al software (Milan, 2013a). De la misma forma, este tipo de activismo requiere un alto grado de especialización y experiencia técnica, que es típicamente premiado con reputación individual y un papel central en cada grupo (Hintz y Milan, 2009); (c.f. Coleman 2013).

\section{Desde las relaciones}

internacionales: las redes de campaña transnacionales como agentes de información

estrategias de campaña de las organizaciones de la socieda dus morales para usar las pruebas estadísticas en sus esfuerzos a favor de sus causas. Mientras que se sabe mucho de la evolución del civismo transnacional en la era de internet (ver, por ejemplo, della Porta y Tarrow, 2005), hasta ahora, la academia no se ha ocupado de la evolución de las estructuras de la sociedad ción con el cambio dramático que están experimentando los flujos globales de información. Sin embargo, estudiosos de las relaciones internacionales como Keck y Sikkink ofrecen herramientas conceptuales, como la noción de las redes de campaña transnacionales (conocidas por sus siglas en inglés, TAN), que son potencialmente útiles a la hora de interpretar las dinámicas relacionadas con datos en la sociedad civil global. Estas son redes de actores ricas en valor, caracterizadas por la producción, intercambio y uso estratégico de la información. Buscan influir sobre los procesos de decisión, pero también "transformar los términos y la naturaleza del debate" (Keck y Sikkink, 2008, p. 2). Las TAN incluyen una vasta muestra de grupos que operan de forma transfronteriza, eperan les. Son particularmente útiles para mapear las emergentes redes de activistas de datos, precisamente porque tienen la información en su centro, en una era donde los big data representan la forma más novedosa que la información está adoptando.

En 2013, el Vicesecretario General del Depar- Los activistas de datos se movilizan a un nivel tamento de Asuntos Económicos y Sociales de los Estados Unidos, Wu Hongbo, reclamó más datos en el desarrollo. "La estadística está dando forma a nuestra comprensión del mundo", dijo ante la Comisión Estadística (UN DESA 2014). Este renovado interés de las organizay la estadística seguramente influirá sobre las exploración, extracción y consumo de combustibles en todo el nacionales adoptan la forma de un TAN, pero tienen como base los datos como moneda de cambio de su activismo. Examinar las TAN de este tipo, nos permite explorar cómo la sociedad civil transnacional interactúa con los datos, mientras que introduce una nueva forma de relación crítica con los datos: aquella de la campaña que está basada en el análisis de datos, pero al mismo tiempo va más allá de relacionarse directamente con instituciones como gobiernos y empresas. Además, observar gobiernos y empresas. Además, observar el activismo de las TAN nos permite prestar atención a la manera como el advenimiento de los big data altera las dinámicas de poder dentro de la sociedad civil transnacional. Finalmente, la literatura académica sobre redes transpara el análisis del activismo de datos de las TAN: aunque estas normalmente representan altos principios asociados con el cambio institucional y político, tienden a ser excluidas de los círculos de decisión política tradicionales (Hintz y Milan, 2009).

\section{Revisar el activismo de datos: una agenda para la investigación}

El activismo de datos proactivo se identifica con un campo emergente de la acción que combina prácticas de comunic tos sociales de la organización colociva información a su nivel mayor de complejidad: los big data. Está caracterizada por una multiplicidad de combinaciones y actores, tanto colectivos como individuales, así como por una gran cantidad de diferentes tácticas de aproxiproactivo van desde el desarrollo de proyectos tecnologicos y plataformas para la manipulación de datos a la visualización de tendencias en los datos con el objetivo de hacer campaña. Tras esta exploración de la literatura relacionada, estamos en posición de revisar y expandir nuestra noción del activismo de datos.

El activismo de datos emerge de la intersección de varios campos de la acción humana, incluyendo tanto el proceso comunicativo (activismo, campañas y sus variantes interesadas en el empoderamiento a través de los medios y la tecnología) y las profesiones relacionadas con la información (análisis de datos y periodismo de investigación). La Figura 1 muestra las interacciones dinámicas entre estos diferentes campos.

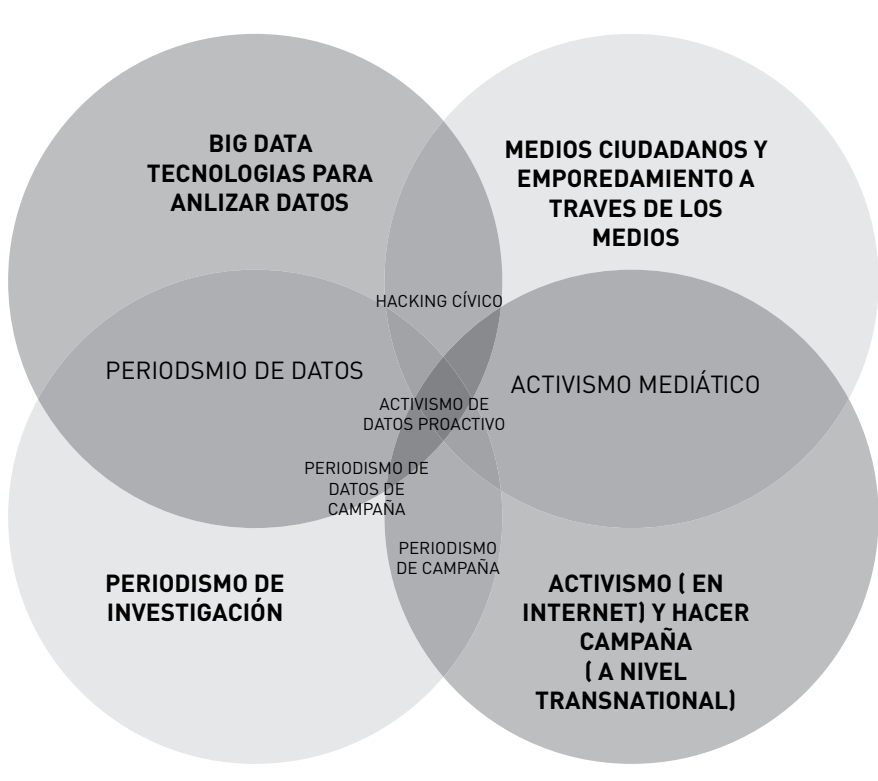

Figura 1. Activismo de datos y campos de acción relacionados.

La Figura 1 puede ser explorada en direcció de las agujas del reloj, empezando por la esfera que engloba los big data, la cual pone en marcha el fenómeno empírico del activismo de datos. Cuando los big data -y las herramientas y 
prácticas del análisis de datos- se encuentran con los medios ciudadanos y otras experiencias de base que ponen en su centro el empoderamiento a través de la relación activa con los medios, emergen casos de "hackeo cívico". los medios, emergen casos de "hackeo civico". Los hackers cívicos son tecnólogos y activistas en favor de los datos abiertos quentarse a los retos relevantes a su comunidad. Así mismo, cuando los medios ciudadanos se encuentran con el activismo, somos testigos del surgimiento del activismo mediático. Por otro lado, cuando el activismo se une a las prácticas y valore periodísticos observamos la aparición del periodismo de campaña. Y cuando los big data se cruzan con el periodismo de investigación, en particular, tenemos el periodismo de datos. Sin embargo, es en la intersección de más de dos esferas donde las tendencias más interesantes comienzan a surgir. Cuando el periodismo de campaña y el periodismo de investigación cruzan sus caminos con los datos y el software para el análisis de datos, tenemos un área de intersección donde aparece el "periodismo de datos de campaña", que combina los valores tradicionales del periodismo con el análisis de datos y la ética del cambio social. Finalmente, en el encuentro entre las cuatro esferas principales, encontramos el activismo de datos proactivo.

¿Qué nociones y mecanismos son aptos para estudiar un campo de acción tan complejo inherentemente interdisciplinario? Desde los estudios del periodismo, hemos aprendido que existen valores como la precisión, el apego a la verdad de los hechos y la ecuanimidad, valores a los que los activistas de datos también se adhieren. De los estudios de los medios de comunicación (alternativos), obtenemos el enfoque en los mecanismos de empoderamiento debido a su capacidad de conectar comunidades, y la noción de activismo como una relación crítica cotidiana con la realidad social circundante
Las relaciones internacionales ofrecen pistas sobre cómo integrar las dinámicas de poder cas redes de activismo de datos emergeno. Le datos emergentes toman la forma de comunidades transfronterizas y deslocalizadas, mientras operan como redes transnacionales de campaña. Parecido a lo que ocurre en otros casos de activismo radical en internet estas "nuevas formas de acción a cociones de sociedad civil tradicio"y y las nociones de socican civil tradicio" y son radicalmentediferentes de la acción colectiva organizada tradicional. Sin embargo, sugerinos llegar al activismo de datos usando las herramientas de los estudios de los movimientos sociales.

Los estudios de los movimientos sociales nos ayudan a entender el activismo de datos como una nueva forma de relación de base con la tecnología, que incluye la acción directa, formas cultures de resistencia, y código. Como en las tácticas de otro tipo de activismo, estas estrategias proporcionan a los activistas una voz moral, que les da la oportunidad de articular y afirmar sus principios y preferencias (Jasper, 1997). Algunos conceptos 1997). Algunos conceptos clave, derivados de la literatura sobre los movimientos sociales, son particularmente útiles para el estudio del activismo de datos: por ejemplo, se puede estudiar la identidad colectiva de los activistas de datos, es decir, la "definición interactiva y compartida" de los individuos que reconocen que comparten ciertas orientaciones y sobre esa base, decidir actuar juntos (Melucci, 1996 , p. 70), preguntando cómo se crea una identidad colectiva sobre la base de una identtécnica. Podemos también investigar las formas organizativas típicas del activismo de datos: ¿Que agrupaciones son más propicias para las actividades y prácticas del activismo de datos? ¿Qué mecanismos internos las regulan? Finalmente, podemos estudiar las relaciones entre los activistas de datos proactivos y las instituciones y normas sociales, incluyendo los gobiernos nacionales y las organizaciones multilaterales.

En conclusión, el activismo de datos puede ser considerado una nueva manifestación de los medios ciudadanos que pone en su mismo centro una perspectiva crítica con respecto a los big data. Como pasa con los medios ciudadanos, el activismo de datos tiene que ver con la política de lo cercano, ya que altera la relación cotidiana entre la ciudadanía y la recolección automatizada de datos. De esa forma, las dimensiones del hacer humano y la política regresan a la maquinaria de recolección de datos. Como ocurre en otros movimientos sociales, los activistas principales tendencias culturales [. ] a través de las cuales nuestras relaciones con el medioambiente normalmente se organizan” (Touraine, 2008, p. 213). Por ello, el activismo de datos probablemente cambiará la forma como la ciudadanía se aproximará a la política computacional y al estado informacional en el futuro, y al mismo tiempo, la forma en la que vemos y practicamos el cambio social. س

\section{Referencias}

Atton, C. (2001). Alternative media. Sage, Thouss Oaks, CA.

Atton, C., \& Hamilton, J.F. (2009). Alternative Journalism. Sage: Thousands Oaks, CA.

Benkler, Y. (2006). The wealth of networks: How social production transforms markets and freedom. New Haven and London: Yale University Press.

Bennett, L.W. \& Segerberg, A. (2012). "The logic of connective action: Digital media and the personalization of contentious politics”. En: Information, Communication \& Society 15 739-768.

Bonechi, M. (2012). Interview with Simon Rogers, editor Guardian Data Blog, London. London. Braman, S. (2009). Change of state: Information, policy, and power. Cambridge: MIT Press

Brevini, B.; Hintz, A., \& McCurdy, P. (2013). Beyond WikiLeaks: implications for the future of communications, journalism and society. Basingstoke, UK: Palgrave Macmillan.

Careless, S. (2000). Advocacy Journalism. The Interim.

Coleman, G. (2013). Coding Freedom: The Ethics and Aesthetics of Hacking. Princeton and Oxford: Princeton University Press.

Cukier, K., \& Mayer-Schoenberger, V. (2013). The Rise of Big Data: How it’s Changing the Way We Think about the World. Foreign Aff. 92, 28-40.

Deibert, R. (2010). After WikiLeaks, a New Era. New York Times. 
Della Porta, D., Tarrow, S. (Eds.) (2005). Transnational protest and global activism. Rowman \& Littlefield, Lanham, MA.

Dickinson, E. (2011). The first Wikileaks revolution? Foreign Policy.

Downing, J.D.H. (2001). Radical Media: Rebellious communication and social movements. Sage: Thousands Oaks, CA.

Dunbar-Hester, C. (2012). "Soldering Toward Media Democracy Technical Practice as Symbolic Value in Radio Activism”. En: Journal of Communication Inquiry 36, 149-169.

Dunbar-Hester, C., 2014. "Producing "Participation”? The Pleasures and Perils of Technical Engagement in Radio Activism”. En: Public Culture 26, 25-50.

Frikel, S., Gross, N. (2005). "A General Theory of Scientific/Intellectual Movements”. En: American Sociological Review, 70, 204-232.

Gangadharan, S. (2012). Digital inclusion and data profiling. First Monday 17.

Garcia, D., Lovink, G. (1997). The ABC of Tactical Media.

Gillmor, D. (2005). The End of Objectivity (Version 0.91).

Gray, J.; Chambers, L. \& Bounegru, L. (Eds.) (2012). The Data Journalism Handbook. O’Reilly, Sebastopol.

Hackett, R.A. \& Carroll, W.K. (2006). Remaking media: the struggle to democratize public communication. London: Routledge, New York.

Hellerstein, J. (2008). "The Commoditization of Massive Data Analysis - Data”. Recuperado de: http://strata.oreilly.com/2008/11/the-commoditization-of-massive.html, consultado el 26 de febrero de 2014

Hess, D.J. (2005). "Technology-and product-oriented movements: Approximating social movement studies and science and technology studies”. En: Science, Technology $\&$ Human Values 30, 515-535.

Hintz, A. \& Milan, S. (2009). "At the margins of Internet governance: grassroots tech groups and communication policy”. En: International Journal of Media \& Cultural Politics 5, 23-38.

Jasper, J., 1997. The Art of Moral Protest: culture, Biography, and Creativity in Social Movements. Chicago: Chicago University Press.

Keck, M. \& Sikkink, K., 2008. Activists beyond borders. Advocacy Networks in International Policy. London: Cornell University Press.

Langlois, A. \& Dubois, F. (Eds.) (2005). Autonomous Media: Activating Resistance \& Dissent. Montréal: Cumulus Press.
Latour, B. (1987). Science in Action: How to Follow Scientists and Engineers through Society. Cambridge, MA: Harvard University Press.

MacKinnon, R. (2012). Consent of the Networked. The Worldwide Struggle for Internet Freedom. New York: Basic Books.

Maxigas. (2014). Internet governance is a trap.

Mehta, A. (2011). Big Data: Powering the Next Industrial Revolution.

Melucci, A. (1996). Challenging Codes. Collective Action in the Information Age. Cambridge, UK: Cambridge University Press.

Meyer, P. (2002). Precision Journalism. A Reporter’s Introduction to Social Science Methods. Rowman \& Littlefield, Lanham, MA.

Milan, S. (2013). Social Movements and Their Technologies: Wiring Social Change. Palgrave Macmillan.

Milan, S. (2013) "WikiLeaks, Anonymous, and the exercise of individuality: Protesting in the cloud”, en: Brevini, B.; Hintz, A., \& McCurdy, P. (Eds.), Beyond WikiLeaks: Implications for the Future of Communications, Journalism and Society. Palgrave Macmillan, Basingstoke, UK, pp. 191-208.

Milan, S., \& Hintz, A. (2013). "Networked Collective Action and the Institutionalized Policy Debate: Bringing Cyberactivism to the Policy Arena?” En Policy \& Internet 5, 7-26.

Milan, S., \& Padovani, C. (2014). "Communication Rights and Media Justice Between Political and Discursive Opportunities: A Historical Perspective”, en: Padovani, C., \& Calabrese, A. (Eds.), Communication Rights and Social Justice: Historical Accounts of Transnational Mobilizations. Palgrave Macmillan, Basingstoke, UK, p. forthcoming.

Northmore, D. (2001). Lifting the Lid: A Guide to Investigative Research. New York: Continuum International Publishing.

Postigo, H. (2012). The digital rights movement the role of technology in subverting digital copyright. Cambridge, Mass: The MIT Press.

Rodriguez, C. (2001). Fissures in the mediascape. An international study of citizens' media. Hampton Press, Cresskill, NJ.

Rosen, J. (2006). Ten Internet Years. Web Users Open the Gates. Washington Post.

Rosen, J. (2008). A Most Useful Definition of Citizen Journalism. PressThink.

Sampedro, V. (2014). El cuarto poder en red. Por un periodismo (de código) libre. Barcelona: Icaria. 
Touraine, A. (2008). “An Introduction to the Study of Social Movements”, en: Ruggiero, V., \&

Montagna, N. (Eds.), Social Movements. A Reader. Routledge, London and New York, pp. 212-217.

Tufekci, Z. (2014). Engeneering the Public: Big data, surveillance, and computational politics. First Monday 19.

UN DESA. (2014). Securing reliable data for development.

Vesset, D.O. (2012). Worldwide Technology and Services: 2012-2016 Forecast (No. 1(238746)), ig Data: Global Overview: Market Analysis. IDC.

Zittrain, J.L. (2008). The Future of the Internet - And How to Stop It. Yale University Press.

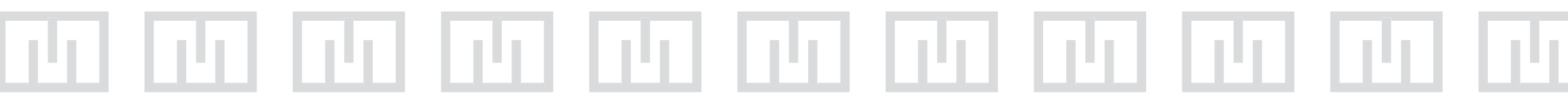

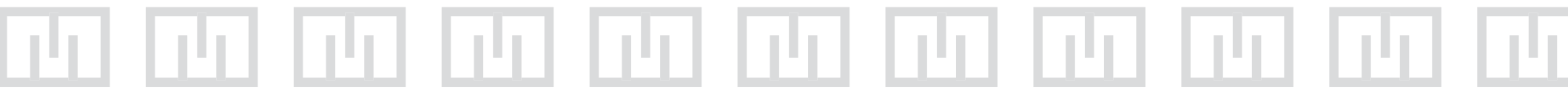

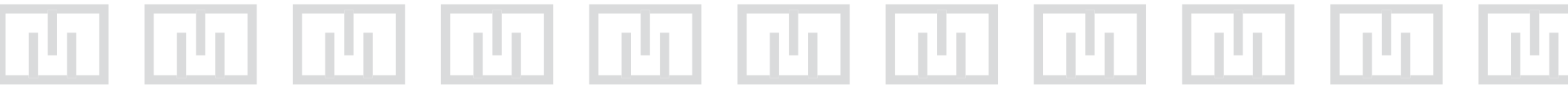

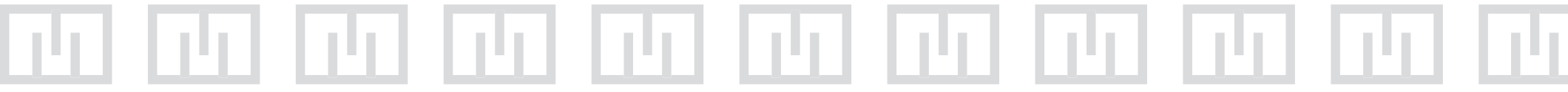

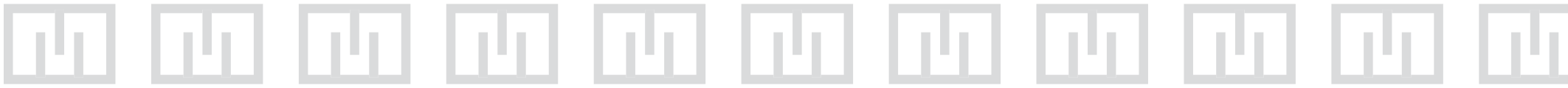

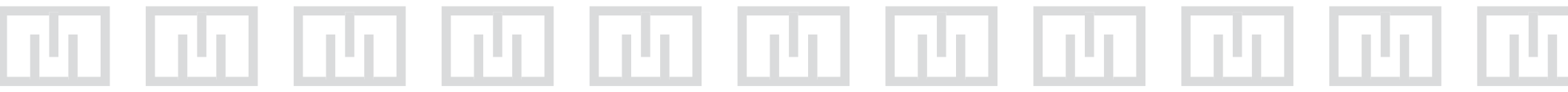

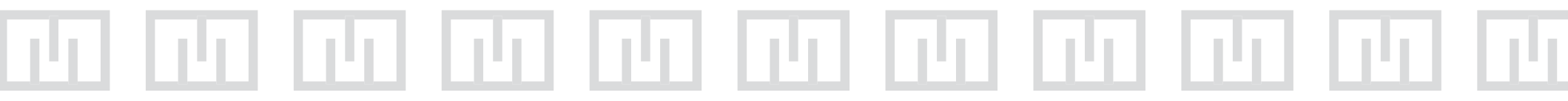

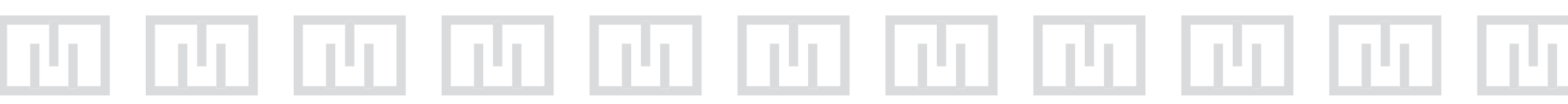

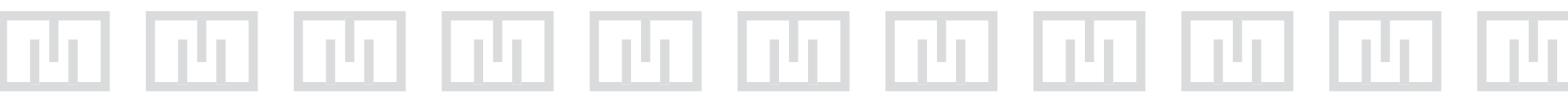

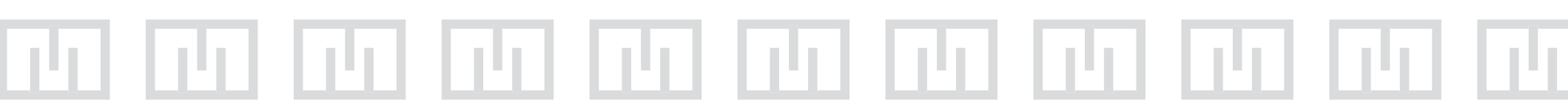

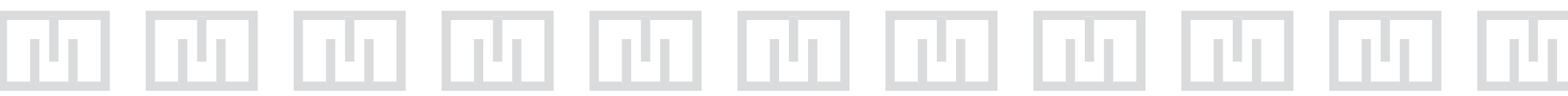

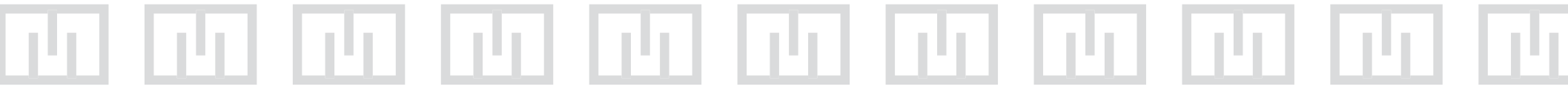

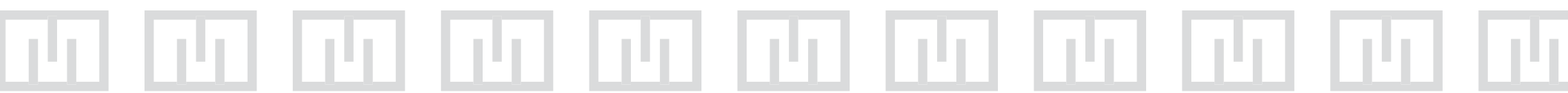

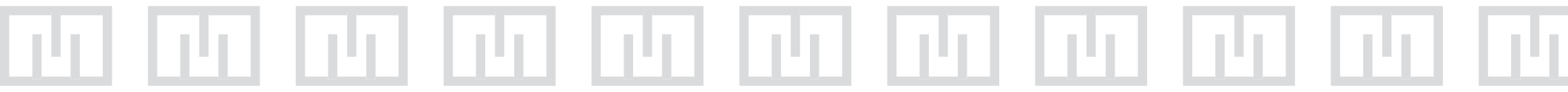

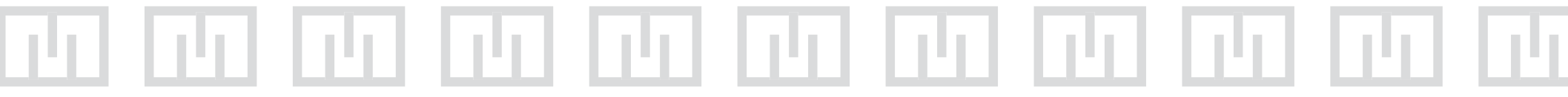

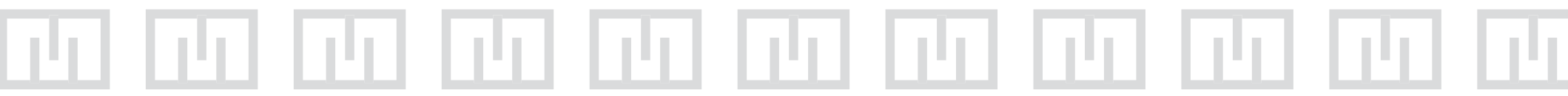

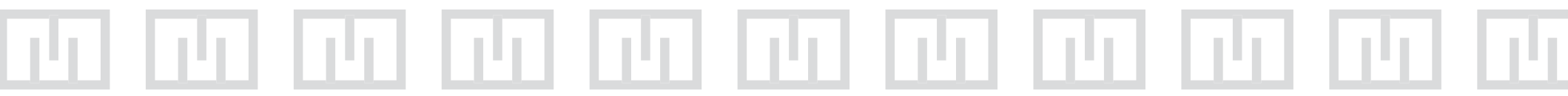

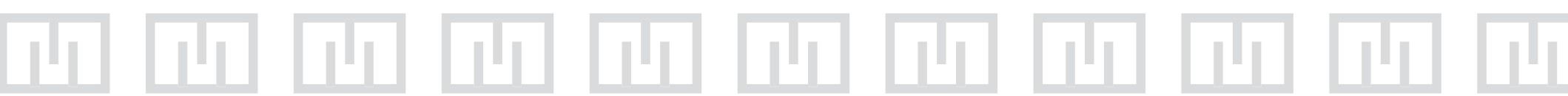

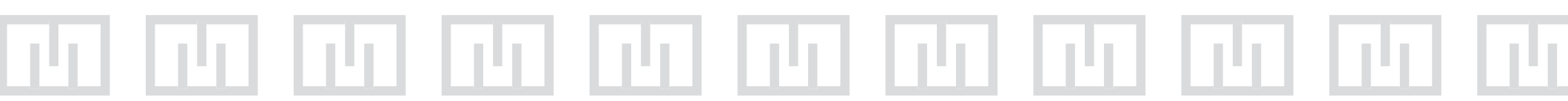

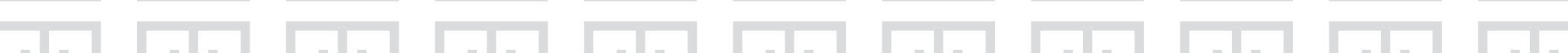

